\title{
Rapidly Progressing Urothelial Carcinoma Due to a Rare TP53 (p.Argl IOPro) Mutation: A Case Report and Review of the Literature
}

\author{
Ragheed Saoud (D)' \\ Thomas H Sanford ${ }^{2}$ \\ Stephen M Hewitt ${ }^{3}$ \\ Andrea B Apolo ${ }^{3}$ \\ Piyush K Agarwal'
}

'Section of Urology, Department of Surgery, The University of Chicago Medicine, Chicago, IL, USA; ${ }^{2}$ Department of Urology, Upstate University Hospital, Syracuse, NY, USA; ${ }^{3}$ Center for Cancer Research, National Cancer Institute, Bethesda, MD, USA
Correspondence: Ragheed Saoud Section of Urology, Department of Surgery, The University of Chicago Medicine, 584I S. Maryland Ave., Rm. J-663, Chicago, IL, 60637, USA

Tel + I 7737029757

Fax +I 7739260732

Email Ragheed.saoud@uchospitals.edu

\begin{abstract}
We present a case of a 69-year-old male patient diagnosed with high grade $\left(\mathrm{T}_{1}\right.$ HG) urothelial carcinoma of the bladder who progressed rapidly towards muscle invasive disease and eventually death despite neoadjuvant chemotherapy and radical cystectomy. We postulate that this may be due to a deleterious underlying somatic gene mutation. Molecular pathologic data obtained on the initial, non-muscle invasive tumor and the final cystectomy specimen, revealed the same TP53 mutation (p.Arg110Pro) in both specimens with a variant allele frequency of $44 \%$. The tumor was tested for 50 common gene mutations in urothelial carcinoma and no other identifiable DNA repair mutations were found, suggesting that this specific TP53 aberration, one that has never been reported in the bladder cancer literature, could be particularly deleterious. Knowing that bladder cancer cell lines that lack TP53 are more resistant to cisplatin and because the tumor lacked any other DNA mutation, this patient may have been a candidate for upfront surgery without neoadjuvant chemotherapy. In addition to histological analysis of the tumor, early molecular and cytogenetic characterization of resected tissue is essential in predicting progression and eventual prognosis of the disease based on identifiable gene mutations. Further comparative prospective studies are required to clarify the importance of molecular heterogeneity and subtyping in bladder cancer.
\end{abstract}

Keywords: invasive, missense mutation, mismatch repair gene

\section{Introduction}

Urothelial carcinoma of the bladder is the ninth most common malignancy worldwide and the second most common urological malignancy after prostate cancer. ${ }^{1}$ It presents in multiple forms ranging from benign polyps to more aggressive tumors with a grave prognosis. We present a patient who progressed rapidly towards death after treatment of muscle invasive disease and postulate that this may be due to a deleterious underlying somatic gene mutation.

\section{Case Description}

A 69-year-old male former smoker presented to clinic with non-specific urinary symptoms in 2015. Cystoscopy revealed bladder tumors, which on transurethral resection revealed high grade $(\mathrm{HG})$ urothelial carcinoma invading the lamina propria (pT1 HG). Follow-up transurethral resection in 2017, revealed muscle invasive disease (pT2) with foci of carcinoma-in-situ (CIS). He underwent neoadjuvant chemotherapy but could not complete the course due to intermittent atrial 


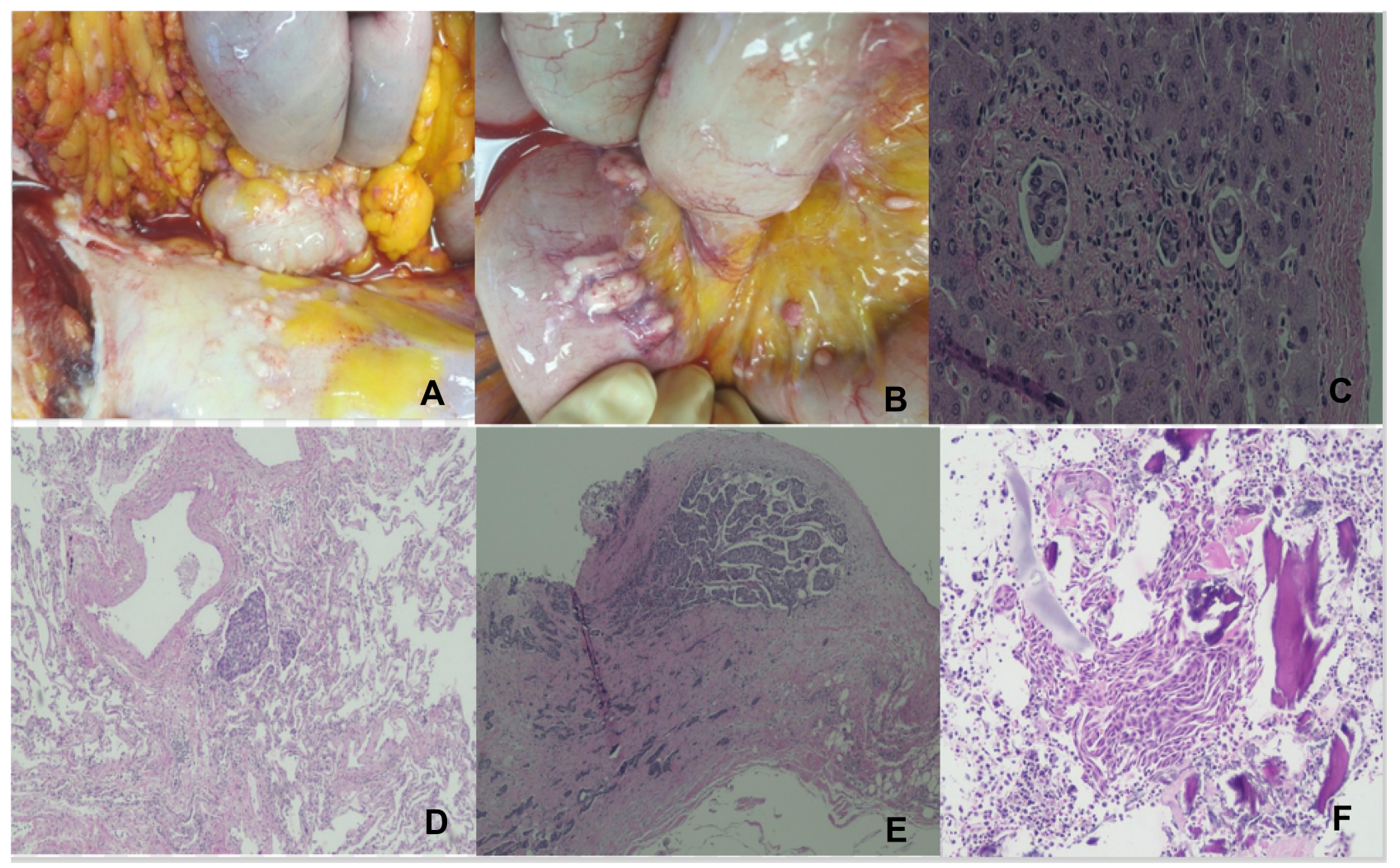

Figure I Axial (A) and coronal (B) CT scans of the abdomen showing abdominal ascites and dilated bowel loops due to obstruction from peritoneal carcinomatosis.

fibrillation. Pre-operative staging PET CT scan with FDG revealed right bladder wall thickness and an avid $1 \mathrm{~cm}$ soft tissue density in the pelvis near the right seminal vesicle suggestive of lymph node involvement. He underwent an open cystoprostatectomy with ileal conduit diversion and extended pelvic lymph node dissection. The specimen was removed en-block with care taken to avoid any tumor spillage into the abdominal cavity. Final pathology revealed muscle-invasive papillary urothelial carcinoma invading the right seminal vesicle with focal micropapillary features. Seven out of 27 lymph nodes were positive for carcinoma, corresponding to pT4aN2R0 disease. The patient was readmitted seven weeks postoperatively due to nonspecific pelvic pain, constipation and an acute rise in serum creatinine. Note was also made of a persistently elevated white count $(11.2-14 \mathrm{~K})$ that had not changed since his diagnosis with CIS three months prior. Crosssectional imaging revealed left hydronephrosis, abdominal ascites, bowel obstruction from peritoneal carcinomatosis (Figure 1A and B), and newly enlarged bilateral inguinal lymph nodes the largest measuring $2.6 \mathrm{~cm}$ in size. The largest intrabdominal mass was biopsied and his abdominal ascites was tapped confirming malignancy. PET/CT scan revealed lesions suspicious of metastatic spread involving the left parietal-temporal skull and 6th ribs (Figure 2A and B). The patient was made nil per os and started on total parenteral nutrition (TPN) prior to placement of a left nephroureteral stent. Hospice was discussed but the patient requested continued and aggressive treatment, prompting the administration of pembrolizumab. In his final week of hospitalization, the patient developed persistent nausea and vomiting requiring a nasogastric tube. He aspirated, progressed towards acute respiratory distress syndrome and ultimately expired three months after surgery. Autopsy revealed metastatic involvement of the diaphragm, small and large bowels, liver, lung, gall bladder, and bone marrow (Figure 3A-F).

\section{Discussion}

The patient's disease exhibited rapid progression after radical cystectomy due to high-grade invasive urothelial carcinoma. Molecular pathologic data obtained on the initial, non-muscle invasive tumor and the final cystectomy specimen, revealed the same TP53 mutation (p. Arg110Pro) in both specimens with a variant allele frequency of $44 \%$. The cancer progressed rapidly without any 


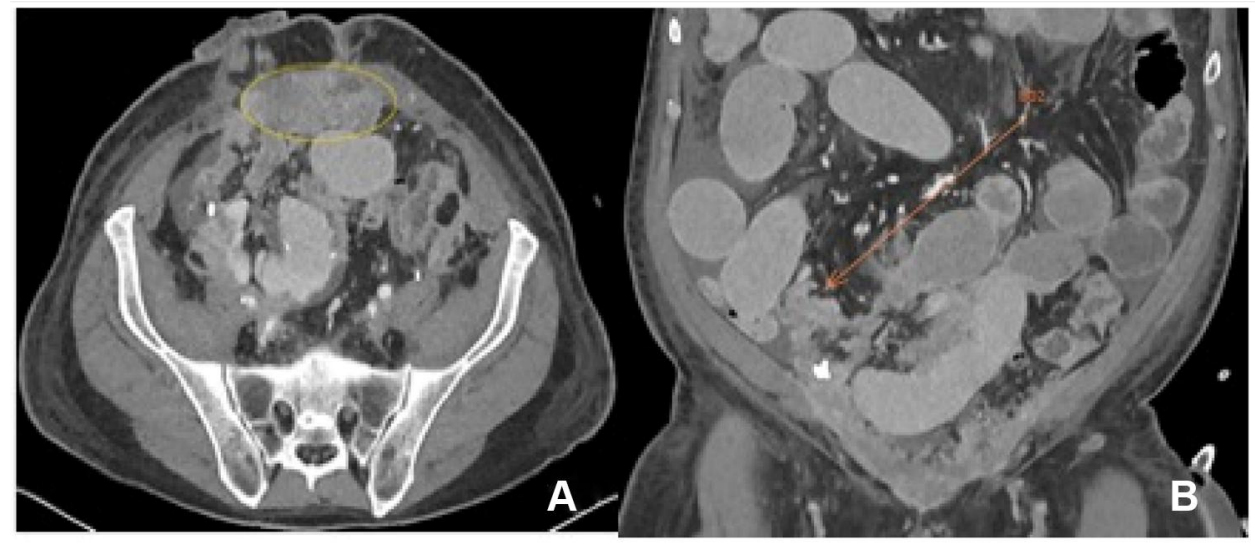

Figure 2 PET NaF showing focal abnormal uptake at $(\mathbf{A})$ the left parietal-temporal skull and (B) the left 6th rib both suspicious for metastatic disease.

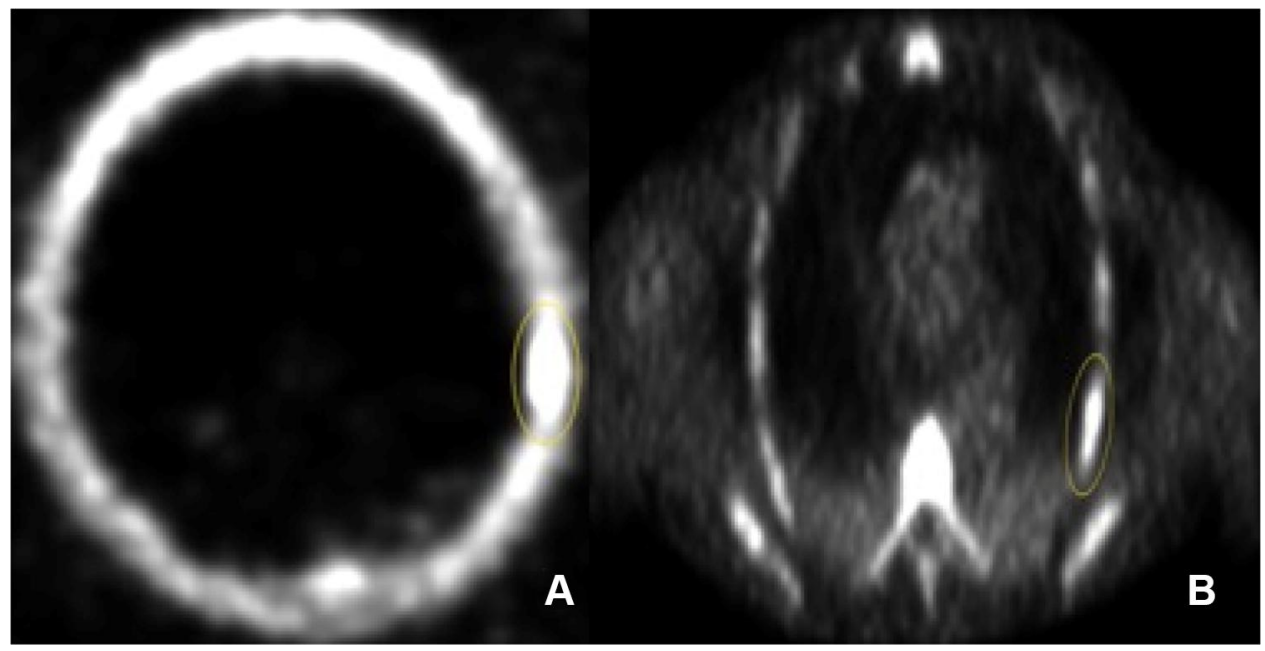

Figure 3 (A-F) Metastatic urothelial carcinoma to the (A) diaphragm (B) small bowel, (C) liver (H\&E stain, original magnification 200x), (D) lung (original magnification 100x), (E) gallbladder (original magnification 40x), and (F) bone marrow (original magnification 200x).

other identifiable DNA repair mutations, suggesting that this specific TP53 aberration could be particularly deleterious. Of note, the patient's white blood cell (WBC) count was elevated before the initiation of neoadjuvant chemotherapy and remained persistently elevated after surgery. This early elevation raises the suspicion of a leukemoid reaction induced by malignant urothelial cells and underlying micrometastatic disease. ${ }^{2}$

We present this case to highlight how even patients with non-muscle invasive (NMIBC) disease may rapidly progress towards metastatic muscle invasive cancer (MIBC) and death. We hypothesize that in addition to histological analysis of the tumor, early molecular and cytogenetic characterization of resected tissue is essential in predicting prognosis of the disease based on identifiable gene mutations.
Molecular characterization is essential for predicting a tumor's response to chemotherapy, be it systemic or intravesical. The tumor was tested for 50 common gene mutations in urothelial carcinoma and was negative for all except TP53 with "R110P" missense mutation. This mutation has never been reported in the bladder cancer literature. We queried 1934 bladder cancer specimens reported in 11 studies in the cBio portal of cancer genomics and none of them displayed this TP53 mutation. However, among 14,126 cancer specimens in the literature, it was found in 10 patients with thyroid, lung, thymic, or breast cancer. Forty percent of these patients died within one year of diagnosis, a pattern similar to our patient's prognosis indicating a potentially deleterious outcome of this mutation. Knowing that bladder cancer cell lines that lack TP53 are more resistant to cisplatin, ${ }^{3}$ and because the tumor 
lacked any other DNA mutation, the benefit of neoadjuvant chemotherapy may have been low. Moreover, such tumors (p53-like) which are infiltrated by a high concentration of lymphocytes and cancer-associated-fibroblasts, usually exhibit cisplatin resistance via upregulation of the ER $\beta / \mathrm{Bcl}-2$ pathway. ${ }^{4}$ A large proportion of NMIBC's are fibroblast infiltrated. This is in contrast to NMIBC's that are especially chemo-sensitive due to DNA mismatch and repair genes (ERCC, ATM, and FANCC), none of which were present in our patient's tumor. This is further proof that various genetic aberrations manifest differently in tumors and influence their behavior.

To validate the concept of heterogeneity, Seiler et al used whole transcriptome profiling in MIBC to show that the basal subtype of tumor cells is the most sensitive to neoadjuvant chemotherapy. ${ }^{5}$ Unlike others, these basal tumors are associated with the most significant improvement in overall survival after chemotherapy, further highlighting the concept that treatment planning should be patient and tumor specific; hence molecular subtyping plays an important role in such decision-making.

\section{Conclusion}

Urothelial carcinoma is a heterogeneous disease that requires treatment based on the stage, grade, and molecular nuances of every tumor. Rapid tumor progression in the presence of a solitary TP53 gene mutation warrants further investigation as to the deleterious nature of this particular gene. Comparative prospective studies are needed to clarify the importance of molecular heterogeneity and subtyping on the diagnosis and treatment planning in bladder cancer.

\section{Data Sharing Statement}

Data to be provided upon request.

\section{Ethics Approval}

This report has received approval for chart review and publication.

\section{Consent to Participate}

Written informed consent for publication of the case details and accompanying images was obtained from the patient's son.

\section{Consent for Publication}

All authors provide consent for publication.

\section{Funding}

There is no funding to report.

\section{Disclosure}

All authors declare no conflicts of interest.

\section{References}

1. Ploeg M, Aben KK, Kiemeney LA. The present and future burden of urinary bladder cancer in the world. World $J$ Urol. 2009;27 (3):289-293. doi:10.1007/s00345-009-0383-3

2. Tachibana M, Miyakawa A, Tazaki H, et al. Autocrine growth of transitional cell carcinoma of the bladder induced by granulocyte-colony stimulating factor. Cancer Res. 1995;55:3438-3443.

3. Mari A, D'Andrea D, Abufaraj M, et al. Genetic determinants for chemo- and radiotherapy resistance in bladder cancer. Transl Androl Urol. 2017;6(6):1081-1089. doi:10.21037/tau.2017.08.19

4. Liu DL, Plimack ER, Hoffman-Censits J, et al. Clinical validation of chemotherapy response biomarker ERCC2 in muscle-invasive urothelial bladder carcinoma. JAMA Oncol. 2016;2:1094-1096. doi:10.1001/ jamaoncol.2016.1056

5. Seiler R, Ashab HAD, Erho N, et al. Impact of molecular subtypes in muscle-invasive bladder cancer on predicting response and survival after neoadjuvant chemotherapy. Eur Urol. 2017;72:544-554. doi:10.1016/j.eururo.2017.03.030

\section{Publish your work in this journal}

Research and Reports in Urology is an international, peer-reviewed, open access journal publishing original research, reports, editorials, reviews and commentaries on all aspects of adult and pediatric urology in the clinic and laboratory including the following topics: Pathology, pathophysiology of urological disease; Investigation and treatment of urological disease; Pharmacology of drugs used for the treatment of urological disease. The manuscript management system is completely online and includes a very quick and fair peer-review system, which is all easy to use. Visit http://www.dovepress.com/ testimonials.php to read real quotes from published authors. 